\title{
CYANOBACTERIA Y CIANOTOXINAS EN UNA LAGUNA SOMERA DEL NORDESTE DE ARGENTINA
}

\author{
Marina E. FORASTIER (1)*; Yolanda ZALOCAR(1); Daniela SEDAN(2) y Darío ANDRINOLO(2)
}

RESUMEN: El objetivo de este trabajo fue analizar las variaciones temporales del fitoplancton e identificar y cuantificar las cianobacterias y cianotoxinas de la laguna Aeroclub $\left(27^{\circ} 28^{\prime} \mathrm{S}-58^{\circ} 43^{\prime} \mathrm{W}\right)$, entre octubre de 2005 y mayo de 2008. La densidad del fitoplancton osciló entre 59 y 7113 ind.ml ${ }^{-1}$ y estuvo representado por ocho grupos taxonómicos, con dominancia de Cyanobacteria $>$ Chlorophyceae $>$ Cryptophyceae. Las cianobacterias predominaron durante la mayor parte del estudio (48\% de los muestreos) con un rango de variación entre 3 (25-10-07) y 6741 ind.ml $^{-1}$ (17-03-06). Su densidad presentó correlación estadísticamente significativa con la temperatura del agua $\left(r_{s}=0,511 ; p<0,05 ; g .1 .=56\right)$. La identificación de cianotoxinas se realizó con material concentrado de Microcystis aeruginosa (Kütz.) Kütz. y M. wesenbergii (Kom.) Kom. Los resultados del análisis en HPLC mostraron cuatro variantes de microcistinas: LR, RR, YR y [D-Leu ${ }^{1}$ LR. Durante el estudio, en tres ocasiones Microcystis ( $M$. aeruginosa $+M$. wesenbergii) superaron el límite máximo establecido por la OMS para un Nivel de alerta I (20000 cél.ml ${ }^{-1}$ ). Debido a la escasa acción antrópica en suelos circundantes de la laguna y, la baja concentración de nutrientes detectada en sus aguas, es posible que las floraciones de cianobacterias no superen este nivel de alerta. Sin embargo, cambios en sus niveles tróficos u otras modificaciones antrópicas podrían alterar el actual equilibrio ecológico.

ABSTRACT: The objective of this study was to analyze the temporal variations of phytoplankton and identify and quantify Cyanobacteria and cyanotoxins of the Aeroclub lagoon $\left(27^{\circ} 28^{\prime} \mathrm{S}, 58^{\circ} 43^{\prime} \mathrm{W}\right)$, between October 2005 and May 2008. Density of phytoplankton ranged between 59 and 7,113 ind.ml ${ }^{-1}$ and was represented by eight taxa, with dominance of Cyanobacteria $>$ Chlorophyceae $>$ Cryptophyceae. Cyanobacteria predominated during most of the study ( $48 \%$ of samples) with a range of variation between 3 (10-25-07) and 6,741 ind.ml ${ }^{-1}$ (03-17-06). Its density showed statistically significant correlation with water temperature $\left(r_{s}=0.511, p<0.05\right.$, d.f. $\left.=56\right)$. The identification of cyanotoxins was performed with concentrated material Microcystis aeruginosa (Kütz.) Kütz. and $M$. wesenbergii (Kom.) Kom. The results of HPLC analysis showed four variants of microcystins LR, RR, YR and [DLeu $^{1}$ ] LR. During the study, three times Microcystis $(M$. aeruginosa $+M$. wesenbergii) exceeded the

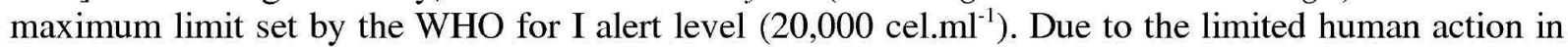
the lagoon surrounding soils and low nutrient concentrations detected in its waters, it is possible that cyanobacterial blooms do not exceed this level of alert. However, changes in their trophic levels or other anthropogenic modifications could alter the current ecological balance.

Palabras claves: Microcystis aeruginosa, $M$. wesenbergii, microcistinas, fitoplancton, cuenca del Riachuelo, provincia de Corrientes, nordeste argentino.

Key words: Microcystis aeruginosa, M. wesenbergii microcystins, phytoplankton, Riachuelo Basin, Corrientes province, northeastern Argentina.

(1) Laboratorio de Ficología. Facultad de Ciencias Exactas y Naturales y Agrimensura (UNNE). Centro de Ecología Aplicada del Litoral (CONICET). Ruta 5, Km 2,5, 3400-Corrientes, Argentina; E-mail: marinaforastier@hotmail.com; zalocaryolanda492@gmail.com.

(2) Laboratorio de Toxicología. Facultad de Ciencias Exactas (UNLP-CONICET), La Plata, Buenos Aires, Argentina. E-mail: danielasedan@yahoo.com.ar; dandrinolo@yahoo.com.

* Autor para correspondencia 


\section{INTRODUCCIÓN}

Los cuerpos de agua continentales generalmente presentan un proceso de eutrofización natural, el que suele ser acelerado por la acción antrópica. Una de las principales consecuencias de este proceso es el incremento de la densidad del fitoplancton, en especial de Cyanobacteria. Ciertas especies en condiciones adecuadas aumentan exponencialmente su biomasa dando lugar a un fenómeno denominado floración (bloom). Las floraciones suelen ser de ocurrencia estacional (durante los meses más cálidos del año) o estar relacionadas a procesos de eutrofización cultural, con sostenido aumento a nivel mundial, y actualmente atribuído al cambio climático global (Paerl and Huisman, 2008, 2009). Estas floraciones alteran el equilibrio del ecosistema acuático, impactando negativamente sobre la calidad del agua (Calijuri et al., 2006).

Las cianobacterias (o "algas verde-azuladas") son organismos procariotas y fotosintéticos. Algunas especies sintetizan cianotoxinas (endotoxinas), las que son liberadas al medio por ruptura de la pared celular, durante el proceso de senescencia natural o por la acción de factores ambientales (Chorus and Bartram, 1999). La persistencia de las cianotoxinas en el agua puede variar desde días hasta años, las que suelen producir efectos sanitarios negativos como así también daños en la salud de la biota acuática, ya que en muchos casos se acumulan en la cadlena trófica (Cazenave et al., 2005; Hudnell, 2008). Las cianotoxinas se clasifican según su modo de acción sobre el órgano blanco del organismo donde actúa, en hepatotoxinas o neurotoxinas, entre otras. Las microcistinas, con más de 80 variantes, son una de las hepatotoxinas de mayor distribución mundial. Un elevado número fue encontrado en diversas reservas de agua utilizadas por animales y seres humanos (Carmichael, 1994). Una alta mortalidad de peces y otros animales (silvestres y domésticos) ha sido frecuentemente reportada (Mancini et al., 2010), sin embargo el evento más relevante que involucró la vida de personas ha sido llamado "el Síndrome Caruarú" que ocurrió con pacientes de hemodiálisis en la ciudad de Caruarú, Brasil (Azevedo et al., 2002).

En Argentina las floraciones de cianobacterias son comunes en ríos y lagunas. Los primeros sucesos fueron descriptos hace más de 90 años por Seckt (1921) y las primeras floraciones tóxicas con muerte de animales vacunos en la provincia de Buenos Aires, fue comprobado treinta años atrás por Odriozola et al. (1984).

Las principales cianotoxinas identificadas en los ríos Paraná, Uruguay y Río de la Plata pertenecen a microcistinas LR, RR, YR y [D-Leu ${ }^{1}$ LR (De León and Yunes, 2001; Andrinolo et al., 2007; Forastier et al., 2010). La última ([D-Leu $\left.{ }^{1}\right]$ LR) es considerada una variante autóctona de la Cuenca del Plata (Rosso et al., 2014).

La región del nordeste argentino se caracteriza por tener un alto número de cuerpos de agua (lagunas, esteros, bañados, arroyos y ríos) en relación al resto de la Argentina. Presenta una rica y variada ficoflora (Tell, 1994; Zalocar de Domitrovic et al., 1998 a, b; Zalocar de Domitrovic, 2003), con elevada densidad y variedad de cianobacterias, citadas como potencialmente tóxicas y/o productoras de toxinas (Zalocar de Domitrovic y Forastier, 2005, 2007; Forastier, 2012).

El objetivo de este estudio fue analizar las variaciones temporales de las cianobacterias que integran el fitoplancton e identificar y cuantificar las cianotoxinas del género 
Microcystis ( $M$. aeruginosa $+M$. wesenbergii) en un cuerpo de agua somero de la cuenca del Riachuelo (provincia de Corrientes) durante dos años y cinco meses de estudio.

\section{MATERIALES Y MÉTODOS}

\section{a) Sitio de estudio}

El estudio se llevó a cabo en la laguna Aeroclub $\left(27^{\circ} 28^{\prime} \mathrm{S}-58^{\circ} 43^{\prime} \mathrm{W}\right)$, ubicada en la cuenca del Riachuelo (afluente del río Paraná). Posee forma redondeada, una superficie de aproximadamente 26 ha y una profundidad media de $3,5 \mathrm{~m}\left(Z_{\max }=5 \mathrm{~m}\right)$. Con escasa vegetación en la zona litoral y macrófitos sumergidos (aproximadamente $40 \%$ del total) con predominio de Potamogeton illinoensis Morong. Su régimen hídrico depende directamente de las precipitaciones, cuyas medias anuales oscilan entre 1100 y $1200 \mathrm{~mm}$. El clima de la región es subtropical de transición, con predominante influencia marítima (Bruniard, 1981). Una completa descripciổn y caracterización limnológica de este cuerpo de agua figura en Matveev et al. (1992) y Zalocar de Domitrovic et al. (1998b).

\section{b) Métodos}

Se estableció un sitio de muestreo en la zona central y más profunda de la laguna, los que tuvieron periodicidad mensual y continuidad desde octubre de 2005 a mayo de 2008, con excepción del año 2006, sin muestreos en los meses de julio, septiembre y diciembre.

Los métodos utilizados para la colecta del fitoplancton siguieron los estándares establecidos por Vollenweider (1974) y Sournia (1978). Para el análisis cualitativo las muestras se concentraron por duplicado con una red de plancton de $25 \mu \mathrm{m}$ de abertura de poro. Una de ellas fue fijada in situ con formaldehído al $4 \%$ y la otra fue transportada refrigerada hasta el laboratorio para su observación al microscopio óptico.

Para el estudio cuantitativo las muestras se obtuvieron sub-superficialmente con botellas de $500 \mathrm{ml}$, fueron fijadas in situ con lugol acético y posteriormente analizadas en microscopio invertido (Utermöhl, 1958; Lund et al., 1958) con una magnificación de $400 \mathrm{X}$.

Con el fin de comparar nuestros resultados con los diferentes niveles de alerta establecidos por la OMS, tomando como referencia el número de células de cianobacterias por mililitro (Chorus and Bartram, 1999), se cuantificaron las células de Microcystis (M. aeruginosa $+M$. wesenbergii) siguiendo las sugerencias de Jardim et al. (2002), utilizando una magnificación de $1000 X$. Durante los recuentos de fitoplancton, además se cuantificaron las colonias de ambas especies de Microcystis utilizando bajos aumentos $(100 \mathrm{X})$.

Las variables ambientales tales como temperatura $\left({ }^{\circ} \mathrm{C}\right)$, transparencia del agua (disco de Secchi), pH (peachímetro Metrohm AG Herisau), conductividad (conductímetro YSI 33 SCT) y concentración de oxígeno disuelto (Oxímetro YSI 54 A), fueron determinadas in situ. Paralelamente se tomaron muestras para análisis de algunos nutrientes (nitritos + nitratos, amonio y ortofosfatos), las que fueron conservadas en frío y oscuri- 
dad hasta su procesamiento, de acuerdo a las técnicas APHA (1990), realizado en los laboratorios de Química del Centro de Ecología Aplicada del Litoral.

Para determinaciones de toxicidad, las muestras a utilizar fueron recolectadas con red de plancton $(25 \mu \mathrm{m})$, filtrando un volumen conocido de agua (60 litros) durante los muestreos de febrero de 2006, enero y septiembre de 2007 y mayo de 2008. La toma de estas muestras se definió cuando se observó (a ojo desnudo) una importante acumulación de colonias (floraciones) próximas a la costa. Las cuatro muestras concentradas del género Microcystis, se mantuvieron en freezer $\left(-20^{\circ} \mathrm{C}\right)$ hasta la extracción de las toxinas. Para extraer las mismas, las muestras fueron sometidas a tres ciclos de congelado/descongelado y luego centrifugadas a $5000 \mathrm{rpm}$ en centrífuga Rolco G20. Los extractos obtenidos fueron inicialmente filtrados por un filtro Osmonic de $0,45 \mu \mathrm{m}$ y finalmente por filtros Sep Pack C 18 previamente activados con metanol (MEOH) 100\%. Las microcistinas fueron eluídas con $\mathrm{MEOH} \mathrm{80 \%} \mathrm{(Andrinolo} \mathrm{et} \mathrm{al.,} \mathrm{2007).}$

Los extractos fueron analizados mediante Cromatografia Líquida de Alta Resolución (Shimadzu, LC-20AT) con detección UV (238 nm) y arreglo de díodos (HPLCDAD), columna C18 (Hyperprep HS, $5 \mu \mathrm{m}$ poro, $150 \times 4,6 \mathrm{~mm}$ ), flujo de $1 \mathrm{ml} \cdot \mathrm{min}^{-1}$. Se empleó un método de gradiente entre la fases móviles A (agua $0,05 \%$ ácido trifluoroacético (TFA)) y B (Acetonitrilo 0,05\% TFA). Las microcistinas fueron identificadas por comparación con estándares analíticos SIGMA Chemicals (St. Louis, MO, USA). [DLeu $^{1}$ ] LR fue purificada de M. aeruginosa cepa CAAT 2003 (Rosso et al., 2014).

\section{RESULTADOS}

\section{a) Variables ambientales}

La temperatura del aire presentó un rango de variación entre 11 y $34^{\circ} \mathrm{C}(25,6 \pm$ $6,29)$. Durante todo el estudio no se detectó estratificación térmica en la columna de agua y la temperatura osciló entre 14 y $30^{\circ} \mathrm{C}(24 \pm 5,24)$. Los valores máximos se registraron durante los meses de verano (enero y febrero de 2007) y los mínimos durante el invierno (julio de 2007). La transparencia del agua, con lecturas del disco de Secchi entre 0,5 y $2,20 \mathrm{~m}(1,4 \pm 0,40)$, fue mínima durante los episodios de floraciones, generalmente ocurridas durante los meses más cálidos. El oxígeno disuelto presentó valores entre 4,27 y $13,75 \mathrm{mg} \mathrm{L}^{-1}(7,35 \pm 1,8)$ con sobresaturación superficial durante la mayor parte de cada año. La conductividad eléctrica fluctuó entre 46 y $128 \mu \mathrm{S} \mathrm{cm}^{-1}(70,5 \pm 19) \mathrm{y}$ el pH en un rango entre 6,9 y 8,2 u $(7,4 \pm 0,27)$.

La concentración de nitrógeno como amonio $\left(\mathrm{N}-\mathrm{NH}_{4}\right)$ y, nitritos+nitratos $\left(\mathrm{NO}_{3}{ }^{-}+\right.$ $\mathrm{NO}_{2}^{-}$) varió en un rango desde no detectable hasta $100 \mu \mathrm{g} \mathrm{L}^{-1}$ de amonio (enero, 2008) y hasta $25 \mu \mathrm{g} \mathrm{L}^{-1}$ de nitritos+nitratos (noviembre, 2005). El fósforo disuelto (como ortofosfatos) no fue detectable durante la mayor parte del estudio y, los valores máximos regis-

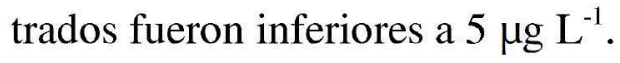




\section{b) Cianobacterias como componentes del fitoplancton}

La densidad del fitoplancton osciló entre 58 (11-10-05) y 7111 ind.ml-1 $(17-03-06)$ con valores promedio de $725( \pm 1288)$ ind. $\mathrm{ml}^{-1}$. Los grupos taxonómicos dominantes en orden decreciente de importancia fueron Cyanobacteria, Chlorophyceae y Cryptophyceae. Dinophyceae y Bacillariophyceae sólo fueron dominantes en un único muestreo (19-12-05 y 25-10-07, respectivamente). Otros grupos como Chrysophyceae, Euglenophyceae y Xanthophyceae estuvieron escasamente representados, con valores inferiores a $5 \%$.

Los valores extremos de abundancia del fitoplancton se observaron a fines de primavera, durante el verano y principios de otoño (máximos) y en invierno (mínimos). Las cianobacterias predominaron durante la mayor parte del período de estudio (48\% de los muestreos) con un rango de variación entre 3 (25-10-07) y 6741 ind.ml ${ }^{-1}$ (17-03-06). La densidad máxima se registró a fines de verano de 2006. En el verano de los años siguientes (2007 y 2008) se redujo a valores inferiores a 1055 ind. $\mathrm{ml}^{-1}$ (Fig. 1).

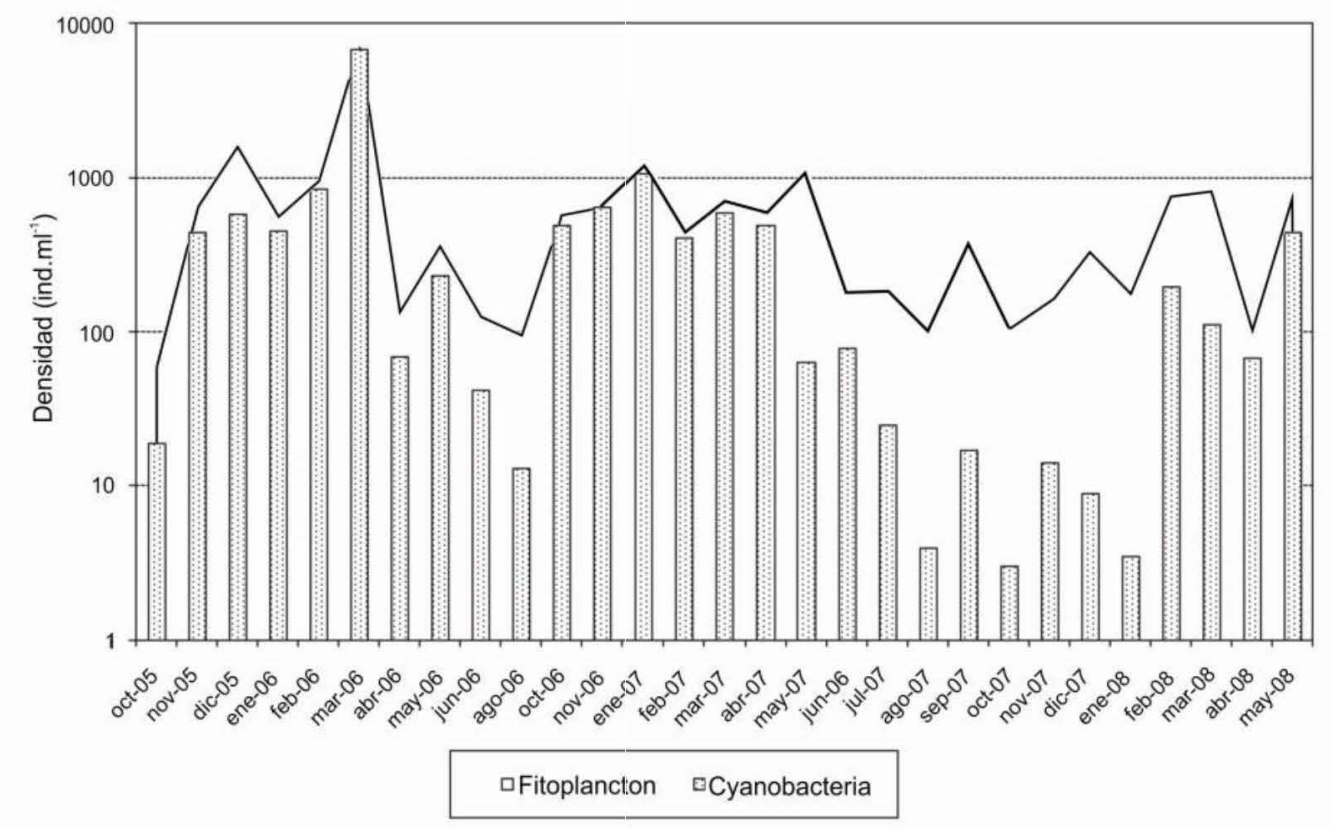

Fig. 1: Variaciones mensuales de Cyanobacteria en relación al fitoplancton total (ind.ml ${ }^{-1}$ ) registradas en la laguna Aeroclub entre octubre de 2005 y mayo de 2008.

Las Oscillatoriales y Nostocales (formas filamentosas) alternaron su dominancia durante los años 2006 y 2007, siendo reemplazadas por Chroococcales coloniales (> 90\%) durante los muestreos de verano de 2008. Las Oscillatoriales estuvieron representadas por Planktolyngbya sp. con valores de densidad máxima de 3740 ind.ml ${ }^{-1}$ (17-0306). Las Nostocales (Cylindrospermopsis raciborskii (Woloszynska) Seenayya et Subba Raju y Aphanizomenon platense Seckt) predominaron en los meses de verano y otoño (2006-2007), superando el 70\% de la densidad total de cianobacterias. La abundancia de

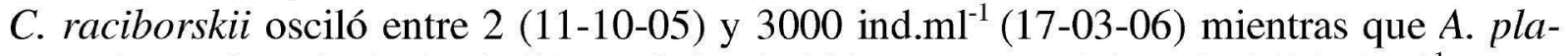

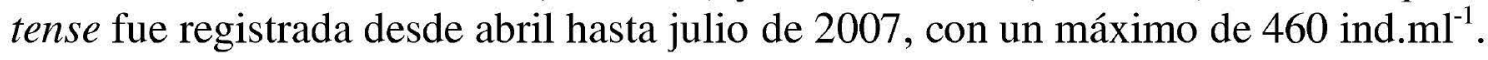


Las Chroococcales (coloniales) estuvieron representadas por Microcystis aeruginosa (máximo 13 colonias.ml ${ }^{-1}$, el 30-10-06) y $M$. wesenbergii (máximo de 7 colonias.ml ${ }^{-1}$, el 22-03-07). También se observaron especies de los géneros Aphanacapsa con valores de 3 a 37 colonias. $\mathrm{ml}^{-1}$ (mayo 2006 y septiembre 2007) y Merismopedia con valores máximos $\left(93\right.$ colonias. $\mathrm{ml}^{-1}$ ) en febrero de 2008.

Las especies del género Microcystis estuvieron presentes durante todo el período de estudio. En algunos muestreos (05-05-06, 30-08-07 y 25-10-07) se registraron por debajo de los límites de detección del método de recuento (Fig. 2) pero fueron observadas en las muestras concentradas con red de plancton.

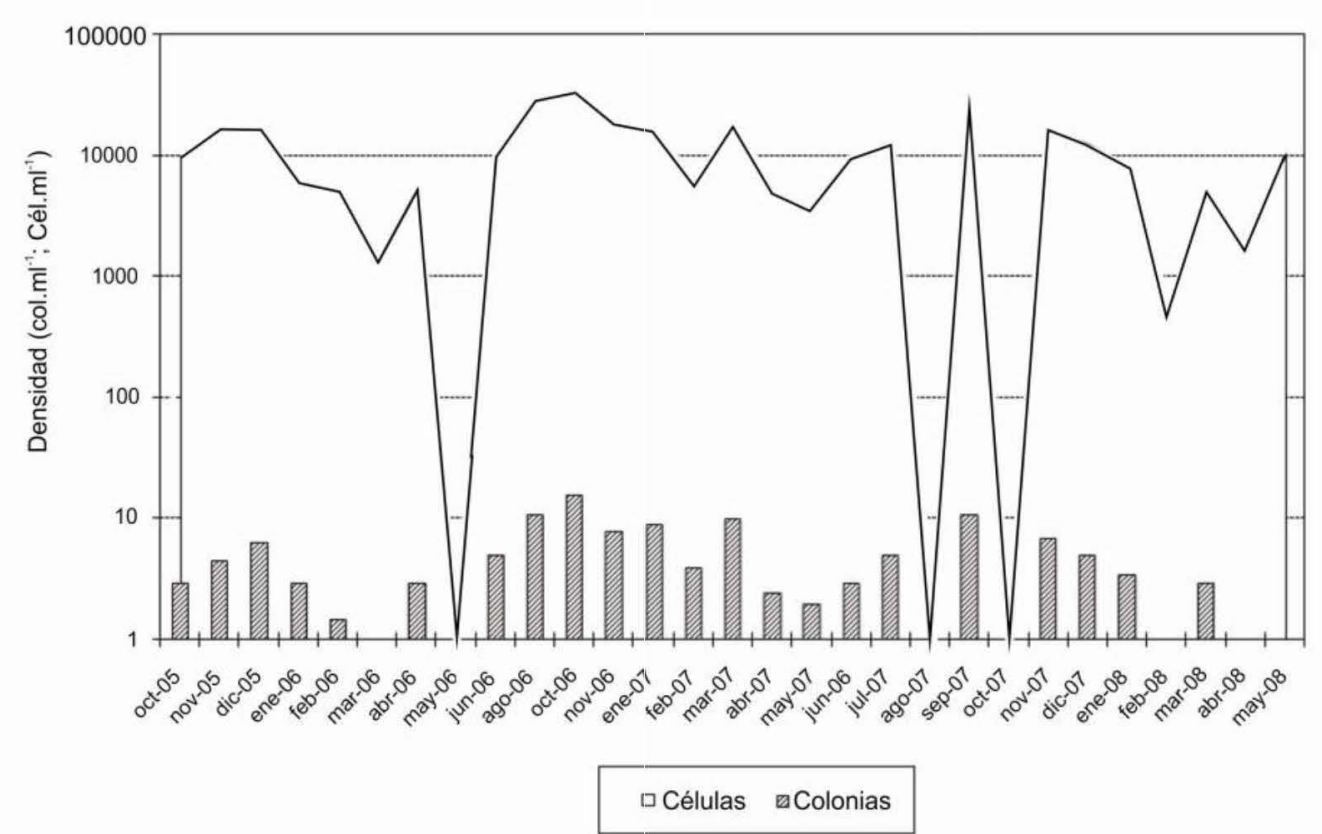

Fig. 2: Variaciones de la densidad de colonias y de células por mililitro de Microcystis aeruginosa + M. wesenbergii registradas durante el estudio.

Con respecto al número de células, $M$. aeruginosa registró un máximo de 26354 (6689 \pm 6018$)$ cél.ml ${ }^{-1}$ en el muestreo del 30-10-06, mientras que $M$. wesenbergii presentó sus máximos el 27-11-06, con 12608 (3362 — 3881) cél.ml ${ }^{-1}$. El número de células de ambas especies (M. aeruginosa $+M$. wesenbergii) fueron superiores a 20000 cél.ml $^{-1}$ (límite máximo establecido por la OMS para el Nivel I de Alerta) durante tres ocasiones, 28440 cél.ml $^{-1}$ el 30-08-06, 32895 cél.ml $^{-1}$ el 30-10-06 y 25785 cél.ml $^{-1}$ el 26-09-07.

La densidad del fitoplancton y de cianobacterias no presentó correlación estadísticamente significativa con las variables físicas y químicas medidas. Sin embargo, una excepción se registró entre la densidad de cianobacterias y la temperatura del agua $\left(\mathrm{r}_{\mathrm{s}}=0,511 ; \mathrm{p}<\right.$ 0,05 ; g.l. = 56). 


\section{c) Cianotoxinas: identificación y cuantificación de microcistinas}

Los resultados del análisis en HPLC de los extractos de cianotoxinas obtenidos durante las floraciones de Microcystis ( $M$. aeruginosa $+M$. wesenbergii) se muestran en los cromatogramas de la Fig. 3. Se identificaron cuatro variantes de microcistinas: LR, RR, YR y [D-Leu $\left.{ }^{1}\right]$ LR. Mcyst-LR estuvo presente en la mayoría de las floraciones detectadas y su concentración osciló entre 0,02 (08-01-07) y $0,4 \mu \mathrm{gL}^{-1}$ (21-02-06). En cambio Mcyst-RR fue la variante con mayor concentración $\left(0,8 \mu \mathrm{gL}^{-1}\right)$ en el verano de 2006 (21-02-06). La Mcyst-YR fue registrada esporádicamente $\left(0,24 \mu \mathrm{gL}^{-1}\right)$. En cambio, [D$\left.\mathrm{Leu}^{1}\right] \mathrm{LR}$ presentó la más baja concentración en las muestras analizadas $\left(0,008 \mu \mathrm{gL}^{-1}\right)$ y fue detectada durante el muestreo del 02-05-08 (Fig. 3).
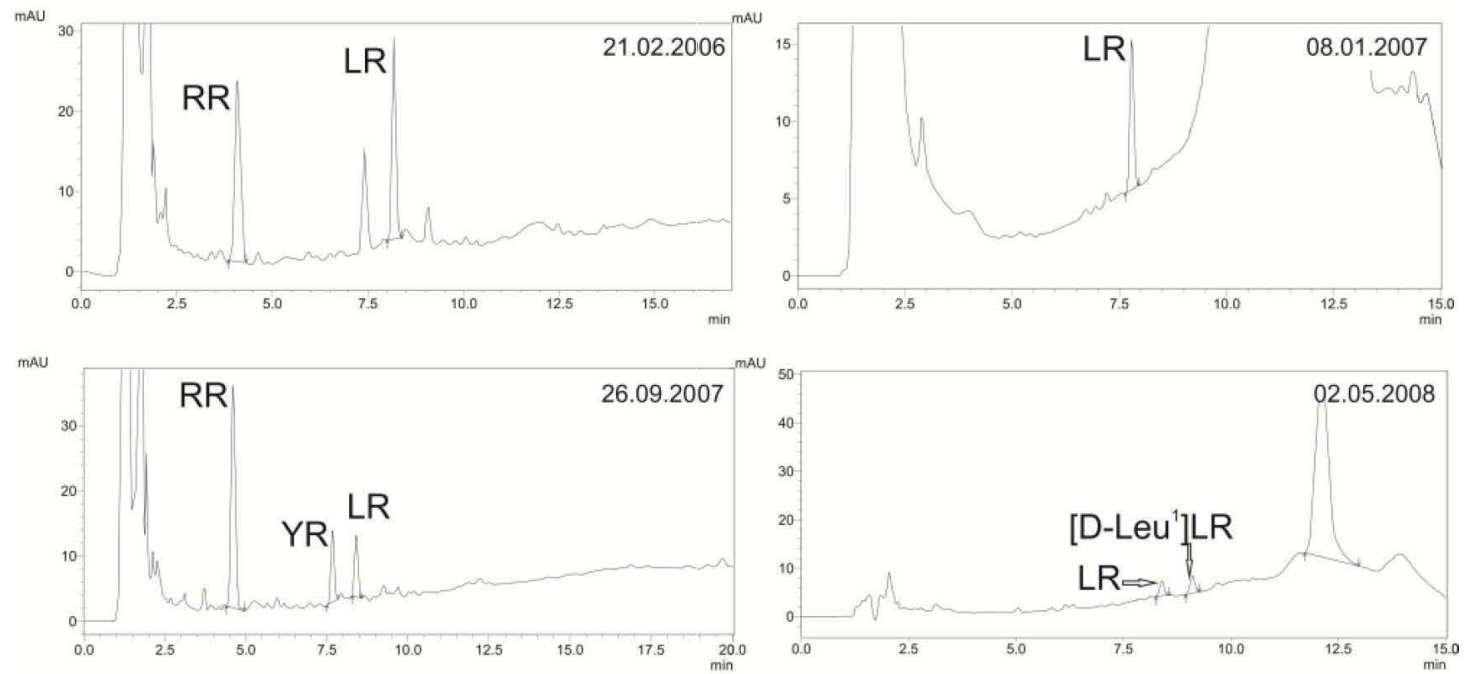

Fig. 3: Cromatogramas (HPLC) de muestras de agua colectadas en la laguna Aeroclub donde se pueden observar las cuatro variantes de microcistinas identificadas (LR, RR, YR y [D-Leu $\left.{ }^{1}\right] \mathrm{LR}$ ).

\section{DiSCUSIÓN}

La composición específica del fitoplancton y los grupos taxonómicos que lo integraron no difirieron mayormente de los resultados obtenidos en trabajos anteriores (Matveev et al., 1992; Zalocar de Domitrovic et al., 1998b), observándose durante este estudio una mayor densidad y frecuencia de floraciones de cianobacterias. Con respecto a las características limnológicas, si bien no presentaron cambios importantes, es de destacar la disminución del nivel hidrométrico, $\mathrm{Z}_{\text {máx }}$ de $6 \mathrm{~m}$ a 4,50-5m, con aparente avance de la vegetación sumergida y mayor transparencia del agua.

El predominio de cianobacterias durante la mayor parte de este estudio, con elevada densidad durante los meses más cálidos (fines de primavera, verano y principios de otoño), estaría asociado a altas temperaturas, tal como fuera demostrado por la correlación positiva entre ambas variables. La temperatura, asociada a la radiación solar serían determinantes de la aparición de cianobacterias y de las variaciones producidas en su densidad durante el ciclo anual (Zębek, 2005, 2006; Reynolds, 2006; Esteves, 2011). En la laguna Aeroclub, estudios realizados durante los años 1995-1996 (Zalocar de Domi- 
trovic et al., 1998b) señalaron dominancia en densidad y biomasa de Cylindrospermopsis raciborskii y Aphanizomenon sp. con incremento de sus poblaciones paralelamente a aumentos de la temperatura del agua y de la radiación solar superiores a $20^{\circ} \mathrm{C}$ y $20 \mathrm{MJ}$ $\mathrm{cm}^{-2}$, respectivamente.

A nivel mundial la frecuencia y duración de las floraciones de cianobacterias están en aumento. Esto ha sido atribuido en parte al calentamiento global (Paerl and Huisman, 2008, 2009) puesto que las cianobacterias crecen a temperaturas óptimas mucho más altas que las algas eucariotas. Varios autores, en base a observaciones de comunidades naturales en el laboratorio y observaciones en el campo han destacado también el efecto positivo de la temperatura sobre el desarrollo de cianobacterias (Reynolds, 2006; De Senerpont Domis et al., 2007; Wagner and Adrian, 2009).

El flujo y disponibilidad de nutrientes son factores importantes que gobiernan la composición y biomasa del fitoplancton en ecosistemas acuáticos (Reynolds, 2006). Históricamente, el nitrógeno $(\mathrm{N})$ y el fósforo $(\mathrm{P})$ fueron señalados como los principales nutrientes limitantes para el crecimiento algal; generalizando, el $\mathrm{N}$ para ambientes tropicales y subtropicales y, el P para ambientes templados. Sus concentraciones frecuentemente suelen ser bajas e insuficientes para el desarrollo celular, no detectándose en la columna de agua, tal como fuera observado en la laguna Aeroclub. La escasez de nutrientes en este cuerpo de agua probablemente se deba a la asimilación por parte de las comunidades fotosintéticas de algas y de macrófitos surnergidos. A pesar de las bajas concentraciones de nutrientes, la densidad de cianobacterias fue elevada, en particular C. raciborskii y $M$. aeruginosa, comparable a lo observado anteriormente en este sitio de estudio (Zalocar de Domitrovic et al., 1998b) y en otras lagunas cercanas pertenecientes a la misma cuenca (Asselborn et al., 1998).

Kosten et al. (2009) en 83 lagos someros de Sud América (entre $5^{\circ}-55^{\circ} \mathrm{S}$ ) no encontraron relación entre densidad de cianobacterias fijadoras (con heterocitos) y no fijadoras de $\mathrm{N}$, con respecto a la concentración de $\mathrm{N}$ inorgánico disuelto. Romo y Villena (2005) demostraron experimentalmente que las cianobacterias pueden predominar en un amplio rango de concentración de nutrientes. Varios autores (Padisák, 1997; Zębek, 2005; Bouvy et al., 2006) destacaron que el efecto de nutrientes sobre el desarrollo de cianobacterias es menos significativo que la influencia ejercida por factores físicos tales como la temperatura, radiación solar y estabilidad de la columna de agua. En investigaciones del fitoplancton de un lago somero de Africa (Senegal) en zona tropical, Bouvy et al. (2006) señalaron que los factores de fuerza que regulan la sucesión del fitoplancton son factores físicos principalmente y, que los niveles de nutrientes deberían ser considerados como un criterio secundario en los ecosistemas acuáticos. Es decir que, el $\mathrm{N}$, el $\mathrm{P}$, ambos o ninguno podrían ser limitantes para el fitoplancton, independientemente de la ubicación geográfica de los sistemas acuáticos (Huszar et al., 2006; Kosten et al., 2009, 2012). Los mencionados autores señalan que el uso de la tierra y la hidrología son factores más importantes que la ubicación geográfica o clima de determinada región. La laguna Aeroclub suele ser utilizada ocasionalmente con fines recreativos por habitantes de propiedades privadas próximas a la misma y no presenta un ingreso importante de nutrientes. 
Las colonias de Microcystis, son de crecimiento lento (no palatables para el zooplancton), acumulan importantes reservas de nutrientes (principalmente fósforo) suelen alcanzar elevada biomasa y persisten durante varias generaciones, aun cuando la concentración de nutrientes no es detectable en ell agua. Tales ventajas competitivas asociadas a las facultades para regular su flotabilidad, por la presencia de aerótopos en las células pueden optimizar su posición en la columna de agua de acuerdo a sus requerimientos nutritivos, favoreciendo su dominancia en etapas avanzadas de la sucesión del fitoplancton (Reynolds, 1972, 2006). Los representantes de este género, particularmente M. aeruginosa y $M$. wesenbergii son cosmopolitas y potenciales productoras de hepatotoxinas (microcistinas).

En la laguna Aeroclub, el número de células que integraron las colonias de $M$. aeruginosa $+M$. wesenbergii no presentaron relación alguna con la concentración de microcistinas detectadas. Esto coincide con lo señalado por la mayoría de los autores, ya que en un ambiente natural pueden co-existir cepas tóxicas y no tóxicas de la misma especie (Dow and Swoboda, 2000; Janse et al., 2004). Además, la producción de toxinas suele ser variable durante las distintas fases de crecimiento (Dyble et al., 2006) y, en algunos casos, su producción puede ser estimulada bajo condiciones ambientales de estrés (Ross et al., 2006).

Las variantes de microcistinas identificadas en este estudio (LR, RR, YR y [D$\left.\mathrm{Leu}^{1}\right] \mathrm{LR}$ ) son de amplia distribución y también fueron registradas en ríos, embalses y lagunas de otras regiones de Argentina (Amé et al., 2003, 2010; Andrinolo et al., 2007; Andrinolo y Caneo, 2009; Ouahid et al., 2011; Rosso et al., 2014). Estas microcistinas, con la excepción de [D-Leu ${ }^{1}$ ] LR, también se encontraron en cuerpos de agua de Chile (Campos et al., 2007). Registros sobre presencia de microcistinas en ambientes leníticos (naturales) de nuestro país, son escasos, debido principalmente a que los estudios se proyectan en embalses de represas de donde se extrae agua para el consumo humano (Pizzolón et al., 1999; Echenique et al., 2006; Echenique y Aguilera, 2009; Ruiz et al., 2009; Mancini et al., 2010; Andrinolo y Ruiz, 2011).

Teniendo en cuenta los Niveles de Alerta determinados por la OMS para cuerpos de agua de uso recreativo, que establece un límite máximo de 20000 cél.ml $^{-1}$ para un nivel de alerta I (Chorus and Bartram, 1999), durante el período estudiado, las cianobac-

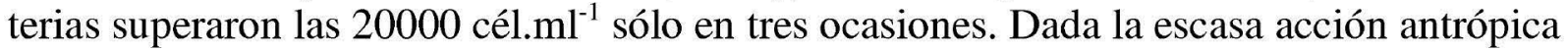
en suelos circundantes a la laguna, sumado a la escasa concentración de nutrientes detectada en el agua, es posible que las floraciones cianobacterianas no superen más que el nivel I de alerta. Sin embargo, cambios en sus niveles tróficos u otras modificaciones antrópicas podrían alterar el actual equilibrio ecológico.

\section{CONCLUSIONES}

Entre los ocho grupos taxonómicos que integraron el fitoplancton de la laguna Aeroclub, las cianobacterias predominaron durante la mayor parte del estudio. Su abundancia (elevada en los meses más cálidos) presentó correlación estadísticamente significativa con la temperatura del agua. Las especies dominantes en densidad fueron Plank- 
tolyngbya sp., Cylindrospermopsis raciborskii y Aphanizomenon platense. En cambio, teniendo en cuenta el número de células predominaron las formas coloniales del género Microcystis.

En este primer estudio de cianotoxinas realizado en ambientes leníticos naturales del nordeste argentino, se identificaron cuatro variantes de microcistinas: LR, RR, YR y [D-Leu $\left.{ }^{1}\right]$ LR, a partir de concentrados de Microcystis aeruginosa $+M$. wesenbergii.

Dada la producción de cianotoxinas por las especies de Microcystis, es recomendable (además de realizar frecuentes monitoreos) no utilizar las aguas de esta laguna para uso recreativo cuando se observan floraciones de cianobacterias.

\section{AGRADECIMIENTOS}

Al personal técnico del CECOAL (UNNE-CONICET), Abel Ramos y Julio Cáceres por el análisis de nutrientes y, a Pablo Bertoni y Casimiro Roberto por su asistencia técnica en los muestreos de campo.

\section{BIBLIOGRAFÍA}

AmÉ, M.V.; M. del PIlar Diaz \& D.A. Wunderlin, 2003. Occurrence of toxic cyanobacterial blooms in San Roque Reservoir (Córdoba, Argentina): a field and chemometric study. Environ. Toxicol., 18: 192-201.

Amé, M.V.; L.N. Galanti; M.L. Menone; M.S. Gerpe; V.J. Moreno \& D.A. Wunderlin, 2010. Microcystin-LR, -RR, -YR and -LA in water samples and fishes from a shallow lake in Argentina. Harmful Algae, 9: 66-73.

Andrinolo, D.; P. Pereira; L. Giannuzzi; C. Aura; S. Massera; M. Caneo; J. Caixach; M. Barco M. \& R. ECHENIQUe, 2007. Ocurrence of Microcystis aeruginosa and Microcystins in Río de La Plata River (Argentina). Acta Toxicol. Argent, 15 (1): 8-14.

ANDRINOLO, D. y M. CANEO, 2009. Efectos sobre la salud humana y animal de cianotoxinas. Mecanismos de acción tóxicas. Pp. 67-78. En: Giannuzzi, L. (ed.): Cyanobacteria y Cianotoxinas. Identificación, Toxicología, Monitoreo y Evaluación de Riesgo. Moglia S.R.L., Corrientes, 237 p.

Andrinolo, D. y M. Ruiz, 2011. Manejo y Control de cianobacterias en lagos, reservorios y ríos. Alertas. Pp.103-116. En: GiannuzzI, L. (ed.): Cianobacterias como determinantes ambientales de la Salud. Buenos Aires: Ministerio de Salud de la Nación, 159 p.

Asselborn, V.M.; Y. Zalocar de Domitrovic y S.L. CAsCo, 1998. Estructura y variaciones del fitoplancton de la laguna Soto (Corrientes, Argentina). Bol. Soc. Argent. Bot., 33 (3-4): 17-27.

APHA (American Public Health Association), 1990. Standard methods of the examination of water and wastewater. APHA, ANWA and WPCF, Washington, 874 p.

Azevedo, S.; W. Carmichael; E. Jochimsen; K. Rinehart; S. Lau; G. Shaw \& G. Eaglesham, 2002. Human intoxication by microcystins during renal dialysis treatment in Caruaru. Brazil. Toxicology, 181-182: 441-446.

Bouvy, M.; N. BA; S. KA; S. SANE; M. PAGANo \& R. ARFi, 2006. Phytoplankton community structure and species assemblage succesión in a shallow tropical lake (Lake Guiers, Senegal). Aquatic Microbial Ecology, 45: 147-161. 
BRUNIARD, E.D., 1981. El clima de las planicies del norte argentino. Facultad de Humanidades de la UNNE, Resistencia (Chaco) Vol. I-II, 379 p. (Tesis doctoral).

Caliunt, M.C.; M.S.A. Alves \& A.C. Alves Dos Santos, 2006. Cianobactérias e cianotoxinas em águas continentais. Rima, São Carlos, 118 p.

Campos, V.; D. Muñoz; M. StTraube; S. LisPerguer y J. Wechesser, 2007. Cianobacterias en cuerpos de agua dulce de la V Region, Chile. Boletín Micológico, 22: 95-100.

CARMichael, W.W., 1994. The toxins of Cyanobacteria. Scientific American, 270 (1): 78-86.

Cazenave, J.; D.A. Wunderlin; M.A. Bistoni; M.V. AmÉ; E. Krause; S. Pflugmacher \& C. Wiegand, 2005. Uptake, tissue distribution and accumulation of Microcystin-RR in Corydoras paleatus, Jenynsia multidentata and Odontesthes bonariensis. A field and laboratory study. Aquat. Toxicol., 75: 178-190.

ChORUs, I. \& BARTRAM, J. (eds.). 1999. Toxic Cyanobacteria in water. A guide to their public health consequences, monitoring and management. WHO. E and FN Spon, London, $416 \mathrm{p}$.

DE LEON, L. \& J.S. YUNES, 2001. First report of a Microcystin-containing bloom of the cyanobacterium Microcystis aeruginosa in the La Plata River, South America. Envir. Toxicol., 16 (1): 110-112.

De Senerpont Domis, L.; W.M. Moou \& J. Huisman, 2007. Climate-induced shifts in an experimental phytoplankton community: a mechanistic approach. Hydrobiologia, 584: 403-413.

Dow, C.S. \& U.K. Swodoba, 2000. Cyanotoxins. Pp. 613-627. En: Witton S.H. \& Potts M. (eds.): The ecology of Cyanobacteria. Their diversity in time and space. Kluwer Academic Publisher, Dordrecht, $666 \mathrm{p}$.

Dyble, J.; P.A. TESTER \& R.W. LiTAKER, 2006. Effects of light intensity on cylindrospermopsin production in the cyanobacterial HAB species Cylindrospermopsis raciborskii. African Journal of Marine Science, 28: 309-312.

Echenique, R.; L. Giannuzzi \& L. FerRari, 2006. Drinking water: problems related to water supply in Bahía Blanca, Argentina. Acta Toxicol. Argent., 14 (2): 2-9.

Echenique, R. y A. Aguilera, 2009. Floraciones de cianobacterias toxígenas en la República Argentina: Antecedentes. Pp. 53-66. En: GiannUZZI, L. (ed.). Cianobacterias y Cianotoxinas: Identificación, toxicología, monitoreo y evaluación. Moglia S.R.L., Corrientes, Argentina, 237 p.

Esteves, F.A. (Ed.), 2011. Fundamentos de limnología. Interciência/FINEP, Río de Janeiro, 790 p.

Forastier, M.E., 2012. Diversidad y toxicidad de Cyanophyta (Cyanobacteria) del nordeste argentino. Tesis doctoral (inédita). Universidad Nacional del Nordeste, Corrientes, Argentina, $218 \mathrm{p}$.

Forastier, M.E.; Y. Zalocar; D. SEDAN \& D. AANDRINOLO, 2010. Occurrence and toxicity of cyanobacterial blooms in Paraná River (Argentina) downstream of the Yacyretá Reservoir. Acta The $8^{\circ}$ international Conference on Toxic Cyanobacteria. Stambul, Turkiye, p. 187.

HudNeLl, H.K. (ed.). 2008. Cyanobacterial Harmful Algal Blooms: State of the Science and Research Needs. Advances in Experimental Medicine and Biology. Vol. 619. Springer 500 p.

HusZAR, V.L.M.; N.F. CARACO; F. ROLAND \& J. COLE, 2006. Nutrient-chlorophyll relationships in tropicalsubtropical lakes: Do temperate models fit? Biogeochemistry, 79: 239-250.

Janse, I.; W. Edwin; A. Kardinaal; M. Meima; J. Fastner; P.M. Visser \& G. Zwart, 2004. Toxic and nontoxic Microcystis colonies in natural populations can be differentiated on the basis of rRNA gene internal transcribed spacer diversity. Applied and Environmental Microbiology, 70 (7): 3979-3987.

JardiM, F.A.; S.O. CAVAlieri; P.C. Gallinari \& L.N.L. Vianna, 2002. Metodologia para a contagem de cianobactérias em células/ml - um novo desafio para o analista de laboratório. Rev. Eng. Sanit. Amb., 7 (3): 109-111. 
Kosten, S.; V.L.M. Huszar; N. Mazzeo; M. Scheffer; L. Da S. Sternberg \& E. Jeppesen, 2009. Lake and watershed characteristics rather than climate influence nutrient limitation in shallow lakes. Ecological Applications, 19: 1791-1804.

Kosten, S.; V.L.M. Huszar; E. Becares; L.S. Costa; E. Van Donk; L.A. Hansson; E. Jeppesen; C. KruK; G. Lacerot; N. Mazzeo; L. De Meester; B. Moss; M. LÜrling; T. Nõges; S. Romo \& M. SCHEFFER, 2012. Warmer climates boost cyanobacterial dominance in shallow lakes. Global Change Biology, 18: 118-126.

Lund, J.W.G.; C. KIPLING \& E.D. Le CREN, 1958. The inverted microscope method of estimating algal numbers and statistical basis of estimating by counting. Hydrobiologia, 11: 143-170.

Mancini, M.; C. Rodriguez; G. Bagnis; A. Liendo; C. Prosperi; M. Bonansea \& J.G. Tundisi, 2010. Cianobacterial bloom and animal mass mortality in a reservoir from Central Argentina. Braz. J. Biol., 70 (3): 841-845.

Matveev, V.; C.C. Martinez; S.M. Frutos \& Y. Zalocar de Domitrovic, 1992. Population control in planktonic crustaceans of a subtropical lake during seasonal succession. Arch. Hydrobiol., 124 (1): 118.

Odriozola, E.; N. Ballabene y A. Salamanco, 1984. Intoxicación en ganado bovino por algas verdesazuladas. Rev. Arg. Microbiol., 16: 219-24.

Ouahid, Y.; M.C. Zaccaro; G. ZulPa; M. Storni; A.M. Stella; J.C. Bossio; M. Tanuz \& F. Del CaMPO, 2011. A single microcystin in a toxic Microcystis bloom from river Río de la Plata (Argentina). Intern. J. Environ. Anal. Chem., 91 (6): 525-536.

PADISÁk, J., 1997. Cylindrospermopsis raciborskii (Woloszynska) Seenayya et Subba Raju, an expanding, highly adaptative cyanobacterium: worldwidw distribution and review of its ecology. Archiv Hydrobiol., 107: 563-593.

Paerl, H.W. \& J. Huisman, 2008. Blooms like it hot. Science, 320: 57-58.

PAERL, H.W. \& J. Huisman, 2009. Climate change: a catalyst for global expansion of harmful cyanobacterial blooms. Environmental Microbiology Reports, 1:27-37.

Pizzolón, L.; B. Tracanna; C. Prósperi and R. Guerrero, 1999. Cyanobacterial blooms in Argentinean inland waters. Lakes \& Reservoirs: Research and Management, 4: 1-5.

REYNOLDS, C.S., 1972. Growth, gas vacuolation and buoyancy in a natural population of planktonic bluegreen algae. Freshwater Biology, 2: 87-106.

REYNOLDS, C.S., 2006. Ecology of phytoplankton. Cambridge University Press, Cambridge, 446 p.

Romo, S. \& M.J. VILLENA, 2005. Phytoplankton strategies and diversity under different nutrient levels and planktivorous fish densities in a shallow Mediterranean lake. J. Plankton Res., 27: 1273-1286.

Ross, C.; L. Santiago-Vasquez \& V. Paul, 2006. Toxin release in response to oxidative stress and programmed cell death in the cyanobacterium Microcystis aeruginosa. Aquatic Toxicology, 78: 6673.

Rosso, L.; D. Sedan; M. Kolman; J. Caixach; C. Flores; J.M. Oteiza; G. Salerno; R. Echenique; L. GiANNUZZI \& D. ANDRINOLO, 2014. Microcystis aeruginosa strain [D-Leu ${ }^{1}$ Mcyst-LR producer, from Buenos Aires province, Argentina. Journal of Coastal Life Medicine, 2 (4): 287-296.

Ruiz, M.; M.I. Rodriguez; A.L. Ruibal; I. Gonzalez; V. Alasia; P. Pellicioni; M. Biagi y D. Lerda, 2009. Exposición al uso y consumo de agua con cianobacterias: efectos en la salud. I Congreso Internacional de Ambiente y Energías Renovables, Villa María, Córdoba, p. 10.

SECKT, H. 1921. Estudios hidrológicos en la Argentina. Schizophyceae. Boletín de la Academia Nacional de Ciencias, 25: 383-429. 
Sournia, A. (ed.), 1978. Phytoplankton Manual. París: UNESCO (United Nations Educational, Scientific and Cultural Organization).

TelL, G., 1994. Planktonic Cyanophyceae from northeastern Argentina. Rev. Hydrobiologia Tropical, 27 (4): 347-360.

UTERMÖHL, H., 1958. Zur Vervollkommnung der quantitativen Phytoplankton-Methodik. Mitteilung Internationale Vereinigung Limnologie, 9: 1-38.

VOLLENWEIDER, R.A. (ed.), 1974. A manual on methods for measuring primary production in aquatic environments. Oxford: Blackwell scientific publications (IBP Handbook $\left.\mathrm{N}^{\circ} 12\right), 225 \mathrm{p}$.

WAGNER C. \& R. AdRIAN, 2009. Cyanobacteria dominance: quantifying the effects of climate change. Limnology and Oceanography, 54: 2460-2468.

Zalocar de Domitrovic, Y., 2003. Fitoplancton de lagunas y cursos de agua del sistema Iberá. Pp. 85-142. En: PoI de NeIFF, A.S.G. (ed.). Limnología del Iberá: Características físicas, químicas y biológicas de las aguas. Ed. Eudene, Corrientes, $191 \mathrm{p}$.

Zalocar de Domitrovic, Y.; S.L. Casco y V.M. Asselborn, 1998. Estudio de la biomasa y diversidad de fitoplancton de la laguna Paiva, Corrientes, Argentina. Physis (Buenos Aires), Sección B, 55 (128129): $1-13$.

Zalocar de Domitrovic, Y.; V.M. Asselborn y S.L. Casco, 1998. Variaciones espaciales y temporales del fitoplancton en un lago subtropical de Argentina. Revista Brasileira de Biología, 58 (3): 359-382.

Zalocar de Domitrovic, Y. \& M.E. Forastier, 2005. Biodiversidad de Cyanophyceae (Cyanobacteria) y especies toxigénicas del litoral fluvial argentino. INSUGEO, Miscelánea, 14: 213-228.

Zalocar de Domitrovic, Y. y M.E. Forastier, 2007. Historia de las floraciones del río Paraná en el tramo argentino: Pp. 1291-1298. En: Feyen J., Aguirre L.F. y Moraes R.M. (eds.): Congreso Internacional sobre Desarrollo, Medio Ambiente y Recursos Naturales: Sostenibilidad a múltiples niveles y escalas. Publicación de la Universidad Mayor de San Simón, Cochabamba-Bolivia, 2033 p.

ZF̨BEK, E., 2005. Qualitative and quantitative changes of blue-green algae in response to physicochemical water parameters in the urban lake Jeziorak Maly. Oceanological and Hydrological Studies, 34: 3956.

ZĘBEK, E., 2006. Quantitative changes ok Planktolyngbya brevicellularis, Limnothrix redekei and Aphanizomenon gracile in annual cycle vs. phycochemical water parameters in the urban lake Jeziorak Maly. Oceanological and Hydrological Studies, 35: 69-84. 